\title{
Chapter 6 \\ Beliefs, Events and Values Inventory \\ Assessment of Global Identity: \\ Implications and Applications \\ for International, Cross-Cultural \\ and Transformative Learning
}

\author{
Felix Wang, Kathleen Pait, Kris Acheson, Lee Sternberger, Renee Staton, \\ and Craig N. Shealy
}

\section{Introduction}

As global communities become more intertwined both technologically and socially, professionals in every field are expected to work with people from different backgrounds and thrive in multicultural settings. Indeed, the international labour market increasingly recognises the need for college graduates to start their professional careers equipped with diverse and open perspectives. Thus, institutions of higher education are under growing pressure from employers to prepare students to be culturally competent and globally minded citizens (Kehl \& Morris, 2008; Trooboff, Vande Berg, \& Rayman, 2008). Fortunately, colleges and universities have not shied away from recognising the importance of global engagement (Altbach \& Knight, 2007). Institutions of higher education around the world are incorporating internationalisation efforts at home and abroad as key components of their strategic planning and curriculum. Due to intentional efforts to support and encourage such engagement, the number of students who participate in an internationalised educational experience during their college career is sharply increasing. In the USA, for example, according to the 2018 Open Doors Report of the Institute of International Education, "the number of international students in the United States surpassed one million for the third consecutive year, increasing by $1.5 \%$ to reach a new high of $1,094,792$ " (IIE, 2018).

\footnotetext{
F. Wang $\cdot$ K. Pait $\cdot$ L. Sternberger $\cdot$ R. Staton $\cdot$ C. N. Shealy $(\bowtie)$

James Madison University, Harrisonburg, VA, USA

e-mail: craigshealy@gmail.com

K. Acheson

Purdue University, West Lafayette, IN, USA
}

J. Frawley et al. (eds.), Cultural Competence and the Higher Education Sector, 
Although we are heartened by such trends-and fully support the purpose and goals of international, cross-cultural and transformative learning experiences (e.g. Altbach \& Knight, 2007; Deardorff, 2016; Kehl \& Morris, 2008; Mezirow, 1997; Taylor \& Cranton, 2012; Trooboff et al., 2008) — our two decades of research strongly indicates that there is no basis for assuming that mere exposure to such "high impact" experiences will be demonstrably salutary for all people, and certainly not in the same way or for the same reasons. That is because the processes and outcomes of international, cross-cultural and transformative learning are determined by context interactions among a range of variables that include, but are not limited to, the learner, the instructor, the experience and other factors (Shealy, 2016; Wandschneider et al., 2015).

As such, a central proposition of the current study is that the impact of international, multicultural and transformative learning must be understood and measured at the level of identity, with all of its depth-based complexity. Although scholars have focused on the construct and development of identity for international students (Kim, 2012) and study abroad students (Hendershot \& Sperandio, 2009), few have examined how the aetiology and structure of identity predispose our students to have differential inclinations and capacities to engage in, and benefit from, such learning experiences in the first place (Acheson, Dirkx, Wiley, Ullum, Kapadia, \& Bhuyan, in press; Spaeth, Schwartz, Nayar, \& Ma, 2016). Through research and understanding of identity's core aspects (e.g. our core needs and internalised beliefs about self, others and the larger world), we begin to understand why, how and under what circumstances "cultural competence" does or does not develop, to what degree, and for whom (Shealy, 2016). Indeed, considerable evidence suggests that we should not devise curricula and implement pedagogies that promote international, crosscultural and transformative learning unless and until we understand how and why our students experience self, others and the larger world as they do, because this affects every aspect of the learning process (e.g. Shealy, in press; Tabit, Legault, Ma, \& Wan, 2016).

\section{Cultural Competence Means More Than Cultural Competence}

Implicit in the above perspective is the following empirically supported contention: it may be necessary, but is by no means sufficient, to include only putative measures of cultural competence to measure cultural competence. Why? Part of the problem is that cultural competence is operationalised according to a very wide array of terms, all of which purport to measure identical or related processes; for example, "intercultural development", "cultural humility", "cultural sensitivity", "ethnocentrism", "multiculturalism" and so on (Deardorff, 2016; Tabit et al., 2016). But the larger problem has to do with the fundamental complexities of being human, in that while we may all be cultural beings, we are not reducible only, or even primarily, 
Table 6.1 Correlation matrix findings above 0.40 between Sociocultural Openness and Beliefs, Events and Values Inventory (BEVI) scales

\begin{tabular}{l|l}
\hline Scales & Correlation \\
\hline Needs closure & -0.90 \\
\hline Ecological resonance & 0.88 \\
\hline Socioemotional convergence & 0.82 \\
\hline Basic closedness & -0.81 \\
\hline Identity diffusion & -0.71 \\
\hline Emotional attunement & 0.77 \\
\hline Socioreligious traditionalism & -0.62 \\
\hline Hard structure & -0.58 \\
\hline Negative life events & -0.57 \\
\hline Divergent determinism & -0.50 \\
\hline
\end{tabular}

to our cultural-beingness. In fact, in terms of predictive validity, our relative degree of "cultural competence"-as measured, for example, by "Sociocultural Openness" and "Global Resonance" on the Beliefs, Events and Values Inventory (BEVI), the central instrument used in the current study-may not even be the best predictor of how we function within a course or programme that is explicitly designed to develop cultural competence (Wandschneider et al., 2015). Rather, other measurable human processes such as Emotional Attunement, Basic Openness, Religious Traditionalism or Self Awareness may, in fact, be more powerfully predictive of "how we do" or "what we learn" in learning experiences than instruments ostensibly designed to facilitate cultural competence (e.g. Giesing, 2017; Iyer, 2013; Tabit et al., 2016; Wandschneider et al., 2015).

Consider, for example, Table 6.1, which presents correlation matrix data to examine the relationship between Sociocultural Openness on the BEVI and other BEVI scales (noting that in this version of the BEVI, informed by exploratory factor analysis, items were allowed to load on different factors in order to help us understand the interrelationship among various scales on the BEVI). ${ }^{1}$ What do such results indicate?

As Wandschneider et al. (2015) note, "individuals scoring high on Sociocultural Openness tend to endorse a worldview that may be characterized as liberal, progressive, accepting, culturally attuned, open, concerned, and globally oriented" (p. 172)—characteristics which are emblematic of "cultural competence" as it is generally understood (e.g. Deardorff, 2016; Tabit et al., 2016). From a review of these correlations in Table 6.1, however, most striking is the empirical fact that individuals who score highly on Sociocultural Openness:

\footnotetext{
${ }^{1}$ Note that a few scales from the EFA "long" version of the BEVI are differently titled from the current BEVI, which is based upon additional factor analytic and other analyses (e.g. Basic Closedness is now Basic Openness; Socio-religious Traditionalism is now Religious Traditionalism; Hard Structure is now Self Certitude; and Divergent Determinism is now Basic Determinism).
} 
- are more likely to indicate that core needs (e.g. for attachment, affection) were met in a "good enough" manner (Needs Closure),

- are more likely to be concerned about or invested in matters that have to do with the environment and natural world (Ecological Resonance),

- appear more able to experience the world in complex "shades of grey", rather than in black and white terms (Socioemotional Convergence),

- are less likely to deny or ignore fundamental thoughts, feelings or needs that generally are experienced as "normative" regarding human existence or functioning (Basic Closedness),

- are less likely to express feelings of confusion and entrapment regarding their current existence and future prospects (Identity Diffusion),

- are more likely to indicate the capacity and inclination to experience affect in self and others, and to value its expression (Emotional Attunement),

- are less likely to endorse a traditional worldview regarding the nature and purpose of religion and its centrality to one's own life (Socioreligious Traditionalism),

- are less likely to express very strong certitude regarding the correctness of one's own way of seeing self, others and the larger world, while denying doubts or weakness (Hard Structure),

- are less likely to report the experience of a high degree of unhappy life experiences during childhood/adolescence or generally in life (Negative Life Events), and

- are less likely to adopt a reflexively contrarian posture regarding the nature of "truth" or "reality" (Divergent Determinism) (p. 172).

The fundamental point-and underlying rationale for this study-is that the concept of culture cannot be understood, and should not be measured, in unidimensional or face valid terms. Again, that is because our "cultural-beingness" is mediated and moderated - that is to say, influenced, shaped and predicted-not only by our beliefs, interests and competencies vis-à-vis different cultures, but by "process variables" such as one's capacity and inclination to experience emotion, or to wonder about self and others, as well as developmental and demographic variables, such as one's life history, ethnicity or gender (e.g. Tabit et al., 2016; Wandschneider et al., 2015). In short, if we really want to understand how and why we are similar and different in our cultural competencies, we have to take these measurable constructs, processes, variables and interactions into account. Ultimately, such complexities are codified within us at any given moment in time not just as our "competencies"cultural or otherwise - but in our fundamental understanding and experience of "why we are who we are", particularly in relation to others and the world at large, which leads us to the multifaceted construct of "global identity", the focus of this chapter.

\section{Global Identity in Theory, Research and Practice}

An important mission of higher education is to address real-life problems through teaching and learning (Keeling, 2006; Cortese, 2003). As Gacel-Ávila (2005) explains, universities should be "a key educational resource for training citizens with 
a critical perspective and the adequate preparation to work and live effectively and successfully in a global context" (p. 125). Examples of such efforts include integration of international and domestic students to enrich "all students learning experience and outcomes", as a model for global citizenship education (Sawir, 2013, p. 371). While the goal of institutions may be the measurement of international learning impact, due to the lack of availability of outcome-based data, institutions may only rely upon output-based data (e.g. the number of individuals studying abroad), which does not provide sufficient information to assess the impact of educational experiences or improve programme quality (Hudzik \& Stohl, 2009). Complicating matters, assessment instruments only collect self-reported and opinion-based data (e.g. asking students if an internationalised learning experience positively impacted their academic engagement), which means that more substantive and compelling data-such as empirically verifiable learning processes and outcomes that illustrate the complex and interacting nature, form and depth of longitudinal change-simply are not measured.

Although internationalised learning experiences are associated with cognitive and emotional change, the larger literature tends to focus on if (or whether) change occurs, rather than examining deeper aspects, such as who we were in the first place, before we engaged in an internationalised educational experience, which interacts further with the experience itself to influence why we change, and to what degree, and under what circumstances. More specifically, international and multicultural education practitioners may focus on curricular and programme design as if such factors alone predicted outcomes. Missing in this assessment calculus is a basic understanding of identity - that is, who students are before they engage in international or multicultural learning programmes - as well as how such factors interact with and influence the results of the entire experience, and also the resulting postprogramme data (Wandschneider et al., 2015). For example, we seldom measure our students' core beliefs, values and life events which serve as the foundation for how they perceive and experience themselves, others and the larger world (Shealy, Sternberger, \& Bhuyan, 2012). However, without a greater appreciation, understanding and assessment of student identity, at the core of which lies the potential for transformative change and learning, international and multicultural courses and programmes may remain desultory and superficial (Spaeth et al., 2016).

Identity is a complex construct because it lies within a dialectic between the structural and performative. Who we believe ourselves to be (avowal) and who others believe us to be (ascription) are functions of forces larger than ourselves and our own agency (Mendoza, Halualani, \& Drzewiecka, 2002). This dialectic creates a dilemma for the term "identity" which may be invoked for a range of purposes, including selfunderstanding (particularistic categorical attributes such as ethnicity or gender); to emphasise cohesion among group members; to highlight aspects of the self that are foundational and unchanging; or, in more postmodern and post-structuralist ways, to highlight the intersubjective process of self-understanding, and the perspectives that emphasise how "self" may be, or become, fluid and fragmented (Brubaker \& Cooper, 2000). 
We conceptualise global identification as one of many expressive processes or functions of the human self and its narrative about itself, which we likewise understand to be our sense of identity. This conceptualisation allows us to acknowledge the power of unconscious and affectively mediated aspects of the self (e.g. our core needs), as well as our ability to reflect upon consciously accessible aspects of the self, as we try to understand who we are, and how and why we change as a result of specific experiences and across our lifetimes (e.g. Aronson, 2012; Ferrari \& Sternberg, 1998; Harter, 1999; Leary \& Tangney, 2012). By examining identity in this way, we shed light not only on the relatively stable structures of beliefs and values that have developed in response to the ways in which our needs have been met over the course of our lives to date, but also on how these systems morph over time in reaction to new experiences (Shealy, 2016; Wandschneider et al., 2015).

\section{Global Identity and the Beliefs, Events and Values Inventory}

Informed by the above theory and research, this study is a multifaceted attempt to examine global identity development in the context of internationalised educational experiences in order to provide recommendations for best practices in international, cross-cultural and transformative programmes and courses. The current study builds upon the work of a multi-year, multi-institution initiative called the Forum BEVI project which brought together thousands of students in the USA and internationally, to examine processes of learning, growth and development. The BEVI is well suited to this type of research and practice because it:

- examines in one broad-spectrum and mixed-methods measure, the interactions between specific relevant mediators (e.g. process variables such as Identity Diffusion, emotional attunement, Sociocultural Openness) and moderators (i.e. class variables such as gender, ethnicity and country of origin that influence the strength and relationship we observe between an intervention and an outcome),

- assesses a comprehensive and empirically derived set of interrelated variables (e.g. including affect, attribution, life history, belief/value structures) that include, but go far beyond, traditional measures of "intercultural competence", which is necessary for the examination of core aspects of the human self and identity,

- links to a depth-based theoretical framework - the Equilintegration (EI) modelthat describes how core human needs interact with formative variables vis-a-vis the internalisation, development and modification of beliefs, values, schemas, attitudes and worldviews, and

- includes an applied report system that facilitates learning, growth and development for individuals, groups and systems.

The construct of global identity is operationalised as an empirically and theoretically derived aggregate score from the BEVI; that is, we are using the Full Scale score on the BEVI as a proxy to represent what we mean by global identity (for more 
information about the Full Scale score, see the "Method" section below). By way of explanation, the Full Scale score represents or captures the "core essence" of what the BEVI measures, including:

\section{basic openness; receptivity to different cultures, religions, and social practices; the tendency (or not) to stereotype in particular ways; self and emotional awareness; and preferred but implicit strategies for making sense of why "other" people and cultures "do what they do". \\ Shealy $(2005$, p. 99)}

As may be clear, then, based upon correlation matrix data (i.e. the statistical interrelationships among scales on a measure) and other statistical methods (e.g. factor analysis, structural equation modelling)_and informed by hundreds of analyses regarding how specific scales are predictive of, or by, a very wide range of formative variables (e.g. gender, ethnicity, education) and outcomes (e.g. grade point average, programme satisfaction) - we have a quite sophisticated understanding of who is, and is not, likely to have the capacity and inclination to engage with cultures that are different from their own (Shealy, 2016; Wandschneider et al., 2015).

Finally, from an analytic perspective, we focus on a new dataset from the BEVI in order to address the following research questions:

1. How are the moderating variables of gender, ethnicity and country of origin associated with an increase in global identity as measured by the BEVI?

2. How are mediating variables (e.g. specific BEVI scales) related to each other and to the BEVI Full Scale score, which operationalises the construct of global identity?

3. What are the applied implications of, and recommendations from, such findings (e.g. how do we use such information to promote global identity development as well as international, multicultural and transformative learning in our students)?

\section{Method}

For this particular study, we accessed a BEVI sample of $N=4926$, which was collected between June 2013 and January 2016. Included in the dataset are participants at a number of higher education institutions who participated in the Forum BEVI project (Wandschneider et al., 2015). As in the original Forum BEVI project, participants were largely undergraduate students at the time of data collection. All were engaged in an internationalised educational experience, such as study abroad, a globalised course on campus or the hosting of an international student. With regard to the moderating variables of gender, ethnicity and country of origin in this study, the analysis included data from 2803 female respondents, 1909 male respondents and 62 respondents of unreported gender. A total of 2599 respondents self-identified as Caucasian and 2,175 identified as non-Caucasian; 3317 respondents were US citizens and 1,457 respondents were other nationalities. There were 4774 participants in total, and 152 participants were excluded from the final sample due to incomplete data (see 
Table 6.2 Sample results for Background-Domain Contrast on the BEVI

\begin{tabular}{|c|c|c|}
\hline Lowest Full Scale & Middle Full Scale & Highest Full Scale \\
\hline Mean $=39 \mid$ Range $=1-51$ & Mean $=60 \mid$ Range $=52-64$ & Mean $=80 \mid$ Range $=65-96$ \\
\hline Background Information & Background Information & Background Information \\
\hline Males: 959 & Males: $\mathbf{5 3 7}$ & Males: 413 \\
\hline Females: 714 & Females: 920 & Females: 1169 \\
\hline Caucasians: 853 & Caucasians: 741 & Caucasians: 1005 \\
\hline Non-Caucasians: 838 & Non-Caucasians: 742 & Non-Caucasians: 595 \\
\hline Details: & Details: & Details: \\
\hline Raised in U.S.: 1088 & Raised in U.S.: 983 & Raised in U.S.: 1246 \\
\hline Not Raised in U.S.: 603 & Not Raised in U.S.: $\mathbf{5 0 0}$ & Not Raised in U.S.: 354 \\
\hline Details: & Details: & - Details: \\
\hline
\end{tabular}

Table 6.2). (For additional information about the demographic characteristics of this sample, and the participants in the Forum BEVI project, see Wandschneider et al., 2015, pp. 156-157.)

The 17 scales of the BEVI are clustered under seven separate domains as follows: I. Formative Variables; II. Fulfilment of Core Needs; III. Tolerance of Disequilibrium; IV. Critical Thinking; V. Self Access; VI. Other Access; and VII. Global Access.

For each administration of the BEVI, a Full Scale score is also generated. To calculate the Full Scale score, 11 of the 17 scales are used. The reasons for including some scales but not others are both empirical, based on correlation matrices and factor analyses, and theoretical, in that inclusion of specific scales across various domains is supported by the EI model that undergirds the BEVI method (Shealy 2016).

Given the empirical and theoretical basis for development of the Full Scale score and its "High", "Medium" or "Low" Optimal derivatives, and in the context of the above discussion of the global identity construct, our analysis uses the BEVI Full Scale score as an indicator of global identity. Overall, a higher or lower score on each of these specific scales results in a higher or lower Full Scale score. For any given group who has taken the BEVI, the highest $30 \%$ of Full Scale scorers are understood, for interpretive purposes, as "High Optimal" scorers; the middle $40 \%$ of Full Scale scorers are understood as "Middle Optimal" scorers; and the lowest $30 \%$ of Full Scale scorers are understood as "Low Optimal" scorers.

The specific scales, as well as their directionality in terms of contributing to a higher or lower Full Scale score, are as follows, noting that in addition to the scale number and name, the designation of "higher" means that a higher score on this specific scale contributes to a higher Full Scale score, while the designation of "lower" means that a lower score on this specific scale contributes to a lower Full Scale score: Scale 3, Needs Fulfilment (Higher); Scale 5, Basic Openness (Higher); Scale 6, Self Certitude (Lower); Scale 7, Basic Determinism (Lower); Scale 10, 
Emotional Attunement (Higher); Scale 11, Self Awareness (Higher); Scale 13, Religious Traditionalism (Lower); Scale 14, Gender Traditionalism (Lower); Scale 15, Sociocultural Openness (Higher); Scale 16, Ecological Resonance (Higher); and Scale 17, Global Resonance (Higher).

Using the Full Scale score as our proxy for "global identity", we examine the relationship between high and low levels of global identity to three moderating (i.e. class or categorical) variables of gender, ethnicity and country of origin. According to Egan and Perry (2001), gender is the "quintessential element" of identity development since it governs all aspects of our lives including self-perception, interactions and relationships with others, and occupation and social status. Gender, one of the moderator variables we examine, demonstrably influences the exploration and reformulation of identity while engaging in international and culturally responsive learning experiences.

A second critical moderator for this study is ethnicity. In selecting this variable, we recognise the complementarity between ethnicity and race. While both are socially constructed, race may have greater connotations of biological difference whereas ethnicity explicitly references cultural differences (Betancourt \& López, 1993). Moreover, since ethnicity is the term used by the BEVI in its collection of demographic data, that term is used for the current study.

Along with gender and ethnicity, we selected country of origin as our third moderating variable of particular interest for obvious reasons; that is, as we are studying the development of global identity, it stands to reason that it would be productive to compare and contrast individuals from different countries of origin. For many students, the act of exploring their national identity (i.e. their country of origin and its attendant culture) is among their most meaningful experiences while abroad (Dolby, 2004).

Overall, this methodological approach allows us to examine and understand how and to what degree these moderating variables are associated with differences among learners in general, and global identity in particular, as measured by the BEVI, even before the learning experience begins. Through this approach, one fundamental contribution such a study makes is illustrating why we must take individual and subgroup differences among our learners into account if we are to understand how "who they are" interacts with "what we do", to produce whatever degree of change we are ultimately able to observe and measure.

The tables and figures in the Results section below represent the Full Scale score comparisons between the highest and lowest 30\%, which are referred to in figures and tables as "top" and "bottom" groups. This comparative technique is an analytic method known as the extreme groups approach (EGA) (Preacher, Rucker, MacCallum, \& Nicewander, 2005). We selected the EGA to ensure that sample characteristics were sufficiently divergent to ascertain valid and reliable findings vis-a-vis the main research questions that comprise this study.

At the macro-level of analysis, we examine interactions between Full Scale scores and the moderators of gender, country of origin and ethnicity and then investigate mediating relationships for each moderator between the Full Scale score and specific individual BEVI scales. Such analysis allows us to explore the complex interactions 
of global identity and global identification with both moderating variables that predict structures of beliefs and values and mediating variables associated with identity development.

At the micro-level of analysis, we home in on specific aspects of global identity, focusing in particular on BEVI Scale 4, Identity Diffusion, which measures the degree to which an individual does not feel a sense of directedness and clarity regarding their own life possibilities. We selected Identify Diffusion as an additional variable precisely because we wondered whether the experience of "otherness", as analysed in this study, might be associated with a greater tendency to experience a painful crisis of identity or a lack of clarity about how to navigate life demands. Here again, we analysed moderators (i.e. categorical variables) of identity using our three distinct formative variables - gender, ethnicity and country of origin - to see whether these categorical variables are related to how, and why, individuals experience their own sense of identity as they do.

\section{Results}

Analysis of results revealed that moderators of identity are strongly associated with the degree to which a global identity emerges. In addition, powerful interactions among BEVI mediators were apparent.

\section{Background-Domain Contrast}

Background-Domain Contrast is an index which illustrates how different or similar the group is, at the level of background information and domain scores, by the lowest 30\%, middle $40 \%$ and highest $30 \%$ of Full Scale scores (see Table 6.2). Background-Domain Contrast is key to understanding whether, and to what degree, group characteristics (i.e. our moderating variables of gender, ethnicity and country of origin) are correlated with Full Scale scores. As shown in Table 6.2, trends emerge for each moderating variable. For example, more males make up the lowest Full Scale score group (959 males as opposed to 714 females), while females far outnumber males in the highest Full Scale score group (1169 females versus 413 males). The number of males decreases consistently from lowest to highest groups (from 959, to 537 , to 413), while the number of females increases consistently (from 714, to 920, to 1169). Table 6.2 provides a broad-stroke view of the data and clear evidence that females are more likely than males to score higher on the Full Scale score. With regard to the other two moderating variables: in general, Caucasians seem more likely 
than non-Caucasians, and people raised in the USA more likely than those raised outside the USA, to score higher on the Full Scale score. ${ }^{2}$

\section{Analysis of BEVI Indexes}

We next analysed several more detailed indexes offered by the BEVI: Aggregate Profile, Decile Profile, Aggregate Profile by Gender, Aggregate Profile by Ethnicity and Aggregate Profile by Country of Origin.

\section{Aggregate Profile}

Aggregate Profile (see Fig. 6.1) offers a comparison of the top and bottom $30 \%$ of the Full Scale scores. In examining Fig. 6.1, we looked for scales with a marked difference between top and bottom Full Scale scores. As one would expect, the top and bottom groups of many scales are very dissimilar in ways that are consistent with emotional intelligence theory and the construction of the Full Scale score on the BEVI (see Table 6.2). For instance, it is clear that participants with the highest Full Scale scores have much higher Needs Fulfilment, Basic Openness, Socioemotional Convergence, Emotional Attunement, Meaning Quest, Sociocultural Openness, Ecological Resonance and Global Resonance.

Because we are operationalising the Full Scale score as a measure of global identity, it is useful to understand to what extent participants are troubled about their sense of identity; therefore, we focus on the Identity Diffusion Scale in Fig. 6.1, for further analysis. It is apparent that the bottom 30\% score for Identity Diffusion is approximately twice as elevated as the top $30 \%$ for this analysis. From an interpretive standpoint, this suggests that individuals in the highest $30 \%$ of the Full Scale score are experiencing approximately half the "Identity Diffusion" of individuals in the lowest $30 \%$ of the Full Scale score. In other words, these results suggest that a greater degree of global identity, as operationalised here, is associated with a substantially lower degree of Identity Diffusion.

Recall from the above scale descriptors that Identity Diffusion measures the degree to which individuals feel a sense of being lost, confused or trapped in their current life situation (Wandschneider et al., 2015). As Spaeth et al. (2016) report, individuals scoring high on Identity Diffusion from the BEVI "tend to be less open or attuned to self, others, and the larger world, less able to tolerate complexity", and are considered

\footnotetext{
${ }^{2}$ The $n$ for a particular group may be lower or higher than expected because a number of respondents may have the same Full Scale score (i.e. distribution rates are calculated on the basis of the percentages of scores that fall within the lowest, middle and highest bands of distribution, rather than on the basis of 1-100, which theoretically is how a Full Scale score could be calculated). Likewise, Decile Profile percentages may shift depending upon the point at which data entry occurs, although the large $\mathrm{N}$ means dispersion patterns will remain consistent.
} 


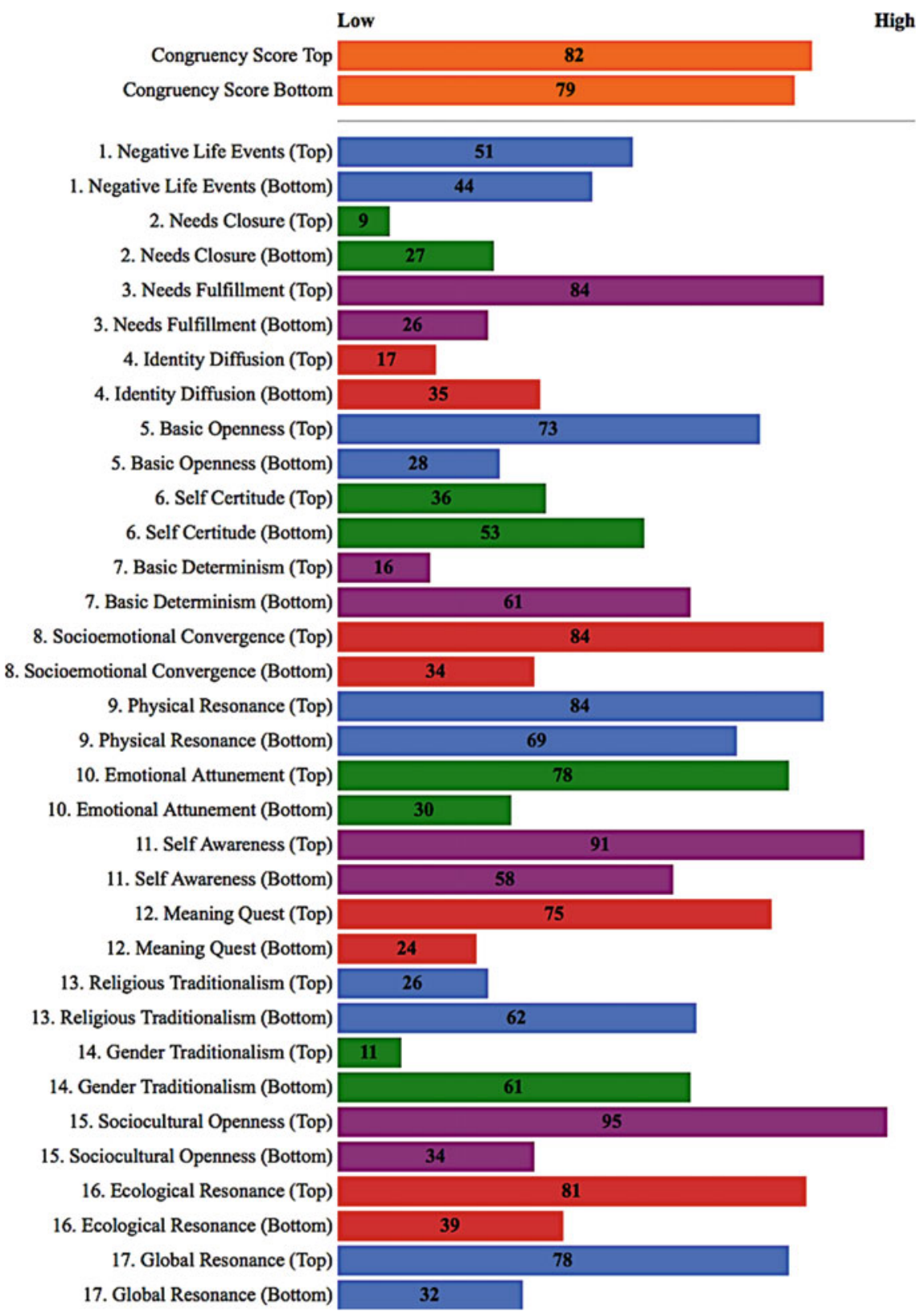

Fig. 6.1 Aggregate Profile-contrast by top 30\% and bottom 30\% of Full Scale scores 
to be more in crisis about their identity, compared to those who scored lower in this particular scale (p. 222). Although it is important to note that neither group from this sample rates very high on Identity Diffusion, the fact that the higher global identity group also demonstrates substantially lower Identity Diffusion offers a measure of validity to our usage of the Full Scale score on the BEVI, since a higher degree of Identity Diffusion should not be associated with a higher degree of global identity; that is, the results are in the direction that would be expected.

\section{Decile Profile}

We next examined Decile Profile to see how individual respondents are dispersed across each of the scales by deciles (i.e. the distribution of scores in 10 "chunks", each representing $10 \%$ of the score range, from the lowest $10 \%$ that individuals may score on each BEVI scale to the highest $10 \%$ ). Note that the darker the grey, the greater the degree of concentration of the overall sample within a particular decile. This index is particularly useful in observing the dispersion of the larger group across all BEVI scales (i.e. it helps illustrate if a group clusters at one or both ends of a scale or is scattered throughout the entire scale). Decile Profile, presented in Fig. 6.2, demonstrates a more nuanced and detailed view of the top and bottom $30 \%$ of Full Scale scores, providing a better understanding of distribution across all BEVI scales including Identity Diffusion.

Consistent with Aggregate Profile, it is clear that Identity Diffusion is concentrated towards the lower deciles for both groups (the top $30 \%$ and bottom $30 \%$ on the Full Scale score). However, note the extensive dispersion for both groups across the entire scale, which indicates that both the top and bottom Full Scale samples do evidence Identity Diffusion. That is one reason why it is important to go beyond mean-based comparisons with assessment, since the variability that may actually exist within a group is masked when we only look at averaged (aggregated) results (Wandschneider et al., 2015).

\section{Aggregate Profile by Gender}

Next, we examined the following three moderator variables (i.e. categorical or class variables, which influence and account for differences we see in mediator variables, such as "Identity Diffusion"): gender, ethnicity and country of origin. Aggregate Profile by Gender compares the scores of females and males across all 17 process scales of the BEVI. More specifically, Aggregate Profile by Gender provides a comparison of how males and females differ at the top and bottom $30 \%$ of the sample. As we would expect to see the given results of Background-Domain Contrast, where we saw that females were more likely to have high Full Scale scores than males, for many scales in Fig. 6.3, females tend to be higher on Basic Openness and Emotional 


\begin{tabular}{|c|c|c|c|c|c|c|c|c|c|c|}
\hline Deciles: & 1 & 2 & 3 & 4 & 5 & 6 & 7 & 8 & 9 & 10 \\
\hline Consistency (Top) & $0 \%$ & $0 \%$ & $0 \%$ & $1 \%$ & $1 \%$ & $3 \%$ & $12 \%$ & $33 \%$ & $36 \%$ & $13 \%$ \\
\hline Consistency (Bottom) & $0 \%$ & $0 \%$ & $2 \%$ & $6 \%$ & $7 \%$ & $18 \%$ & $27 \%$ & $29 \%$ & $9 \%$ & $0 \%$ \\
\hline Congruency (Top) & $0 \%$ & $0 \%$ & $0 \%$ & $0 \%$ & $0 \%$ & $2 \%$ & $7 \%$ & $21 \%$ & $39 \%$ & $31 \%$ \\
\hline Congruency (Bottom) & $0 \%$ & $0 \%$ & $0 \%$ & $0 \%$ & $1 \%$ & $5 \%$ & $13 \%$ & $23 \%$ & $33 \%$ & $25 \%$ \\
\hline 1. Negative Life Events (Top) & $11 \%$ & $16 \%$ & $11 \%$ & $6 \%$ & $7 \%$ & $12 \%$ & $4 \%$ & $9 \%$ & $11 \%$ & $15 \%$ \\
\hline 1. Negative Life Events (Bottom) & $11 \%$ & $14 \%$ & $12 \%$ & $8 \%$ & $9 \%$ & $16 \%$ & $6 \%$ & $9 \%$ & $9 \%$ & $6 \%$ \\
\hline 2. Needs Closure (Top) & $57 \%$ & $11 \%$ & $8 \%$ & $5 \%$ & $5 \%$ & $2 \%$ & $3 \%$ & $2 \%$ & $3 \%$ & $3 \%$ \\
\hline 2. Needs Closure (Bottom) & $28 \%$ & $14 \%$ & $11 \%$ & $11 \%$ & $8 \%$ & $4 \%$ & $8 \%$ & $5 \%$ & $8 \%$ & $3 \%$ \\
\hline 3. Needs Fulfillment (Top) & $0 \%$ & $0 \%$ & $0 \%$ & $1 \%$ & $2 \%$ & $6 \%$ & $10 \%$ & $20 \%$ & $27 \%$ & $33 \%$ \\
\hline 3. Needs Fulfillment (Bottom) & $13 \%$ & $20 \%$ & $24 \%$ & $19 \%$ & $11 \%$ & $6 \%$ & $5 \%$ & $2 \%$ & $0 \%$ & $0 \%$ \\
\hline 4. Identity Diffusion (Top) & $36 \%$ & $17 \%$ & $16 \%$ & $0 \%$ & $12 \%$ & $0 \%$ & $0 \%$ & $9 \%$ & $5 \%$ & $4 \%$ \\
\hline 4. Identity Diffusion (Bottom) & $19 \%$ & $17 \%$ & $18 \%$ & $0 \%$ & $17 \%$ & $0 \%$ & $0 \%$ & $11 \%$ & $8 \%$ & $10 \%$ \\
\hline S. Basic Openness (Top) & $1 \%$ & $5 \%$ & $2 \%$ & $7 \%$ & $5 \%$ & $8 \%$ & $17 \%$ & $10 \%$ & $24 \%$ & $21 \%$ \\
\hline 5. Basic Openness (Bottom) & $15 \%$ & $19 \%$ & $9 \%$ & $20 \%$ & $9 \%$ & $10 \%$ & $11 \%$ & $2 \%$ & $3 \%$ & $1 \%$ \\
\hline 6. Self Certitude (Top) & $20 \%$ & $12 \%$ & $7 \%$ & $18 \%$ & $6 \%$ & $7 \%$ & $6 \%$ & $9 \%$ & $7 \%$ & $7 \%$ \\
\hline 6. Self Certitude (Bottom) & $5 \%$ & $6 \%$ & $5 \%$ & $18 \%$ & $12 \%$ & $12 \%$ & $13 \%$ & $14 \%$ & $8 \%$ & $8 \%$ \\
\hline 7. Basic Determinism (Top) & $32 \%$ & $24 \%$ & $20 \%$ & $7 \%$ & $5 \%$ & $4 \%$ & $3 \%$ & $2 \%$ & $2 \%$ & $1 \%$ \\
\hline 7. Basic Determinism (Bottom) & $3 \%$ & $6 \%$ & $12 \%$ & $9 \%$ & $11 \%$ & $12 \%$ & $10 \%$ & $9 \%$ & $14 \%$ & $13 \%$ \\
\hline 8. Socioemotional Convergence (Top) & $0 \%$ & $0 \%$ & $1 \%$ & $2 \%$ & $4 \%$ & $6 \%$ & $8 \%$ & $18 \%$ & $24 \%$ & $36 \%$ \\
\hline 8. Socioemotional Convergence (Bottom) & $8 \%$ & $15 \%$ & $22 \%$ & $15 \%$ & $11 \%$ & $11 \%$ & $6 \%$ & $7 \%$ & $3 \%$ & $1 \%$ \\
\hline & $0 \%$ & $0 \%$ & $0 \%$ & $0 \%$ & $1 \%$ & $3 \%$ & $5 \%$ & $21 \%$ & $46 \%$ & $23 \%$ \\
\hline 9. Physical Resonance (I & $0 \%$ & $1 \%$ & $2 \%$ & $3 \%$ & $6 \%$ & $14 \%$ & $14 \%$ & $35 \%$ & $23 \%$ & $3 \%$ \\
\hline 10. Emotional Attuneme & $1 \%$ & $1 \%$ & $2 \%$ & $6 \%$ & $5 \%$ & $14 \%$ & $8 \%$ & $16 \%$ & $24 \%$ & $23 \%$ \\
\hline 10. Emotional Attu & $16 \%$ & $11 \%$ & $16 \%$ & $20 \%$ & $8 \%$ & $17 \%$ & $5 \%$ & $5 \%$ & $1 \%$ & $0 \%$ \\
\hline 11. Self Awareness (Top) & $0 \%$ & $0 \%$ & $0 \%$ & $0 \%$ & $0 \%$ & $1 \%$ & $7 \%$ & $6 \%$ & $28 \%$ & $57 \%$ \\
\hline 11. Self Awareness (Bottom) & $2 \%$ & $4 \%$ & $3 \%$ & $13 \%$ & $11 \%$ & $14 \%$ & $29 \%$ & $9 \%$ & $13 \%$ & $3 \%$ \\
\hline 12. Meaning Quest (Top) & $1 \%$ & $1 \%$ & $7 \%$ & $7 \%$ & $8 \%$ & $9 \%$ & $8 \%$ & $19 \%$ & $19 \%$ & $22 \%$ \\
\hline 12. Meaning Quest (Bottom) & $23 \%$ & $10 \%$ & $26 \%$ & $14 \%$ & $8 \%$ & $6 \%$ & $4 \%$ & $6 \%$ & $2 \%$ & $0 \%$ \\
\hline 13. Religious Traditionalism (Top) & $25 \%$ & $21 \%$ & $13 \%$ & $7 \%$ & $12 \%$ & $5 \%$ & $8 \%$ & $4 \%$ & $3 \%$ & $4 \%$ \\
\hline 13. Religious Traditionalism (Bottom) & $5 \%$ & $4 \%$ & $4 \%$ & $5 \%$ & $16 \%$ & $9 \%$ & $17 \%$ & $17 \%$ & $11 \%$ & $12 \%$ \\
\hline 14. Gender Traditionalism (Top) & $45 \%$ & $20 \%$ & $11 \%$ & $6 \%$ & $5 \%$ & $6 \%$ & $2 \%$ & $3 \%$ & $1 \%$ & $1 \%$ \\
\hline 14. Gender Traditionalism (Bottom) & $2 \%$ & $5 \%$ & $8 \%$ & $7 \%$ & $8 \%$ & $19 \%$ & $11 \%$ & $17 \%$ & $11 \%$ & $10 \%$ \\
\hline 15. Sociocultural Openness (Top) & $0 \%$ & $0 \%$ & $0 \%$ & $0 \%$ & $0 \%$ & $1 \%$ & $3 \%$ & $7 \%$ & $15 \%$ & $73 \%$ \\
\hline 15. Sociocultural Openness (Bottom) & $10 \%$ & $12 \%$ & $21 \%$ & $10 \%$ & $18 \%$ & $12 \%$ & $7 \%$ & $5 \%$ & $3 \%$ & $1 \%$ \\
\hline 16. Ecological Resonance (Top) & $1 \%$ & $1 \%$ & $2 \%$ & $3 \%$ & $7 \%$ & $10 \%$ & $13 \%$ & $13 \%$ & $14 \%$ & $36 \%$ \\
\hline 16. Ecological Resonance (Bottom) & $12 \%$ & $8 \%$ & $10 \%$ & $13 \%$ & $16 \%$ & $16 \%$ & $13 \%$ & $5 \%$ & $2 \%$ & $3 \%$ \\
\hline 17. Global Resonance (Top) & $0 \%$ & $1 \%$ & $2 \%$ & $5 \%$ & $0 \%$ & $7 \%$ & $10 \%$ & $15 \%$ & $43 \%$ & $16 \%$ \\
\hline 17. Global Resonance (Bottom) & $9 \%$ & $21 \%$ & $24 \%$ & $18 \%$ & $0 \%$ & $12 \%$ & $8 \%$ & $5 \%$ & $4 \%$ & $1 \%$ \\
\hline Deciles: & 1 & 2 & 3 & 4 & 5 & 6 & 7 & 8 & 9 & 10 \\
\hline Deciles: & 1 & 2 & 3 & 4 & 5 & 6 & 7 & 8 & 9 & 10 \\
\hline
\end{tabular}

Fig. 6.2 Decile Profile—contrast by top 30\% and bottom 30\% of full Scale scores 


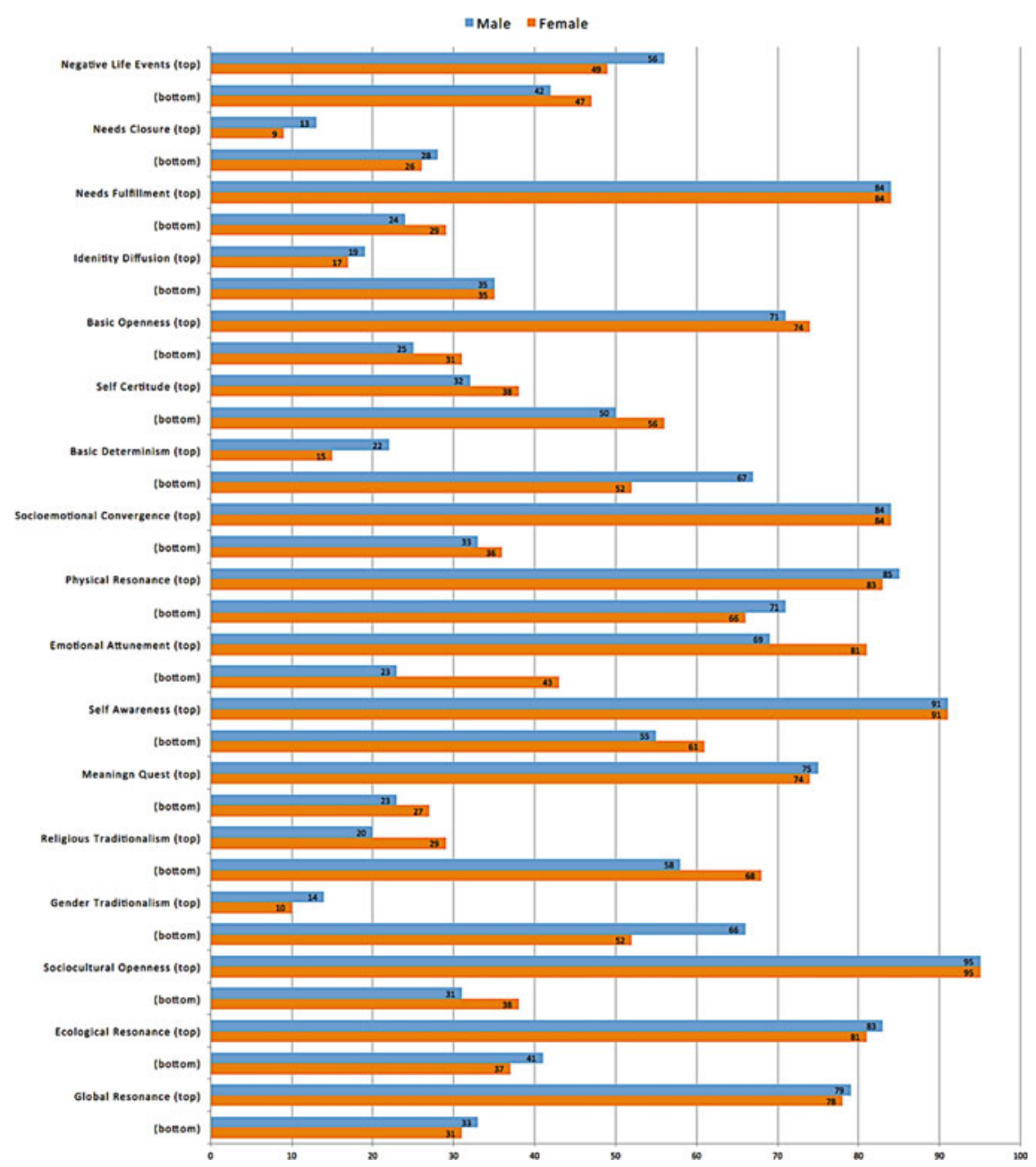

Fig. 6.3 Aggregate Profile—contrast by gender

Attunement and lower on Basic Determinism and Gender Traditionalism. These trends do not hold for all scales. For Ecological Resonance and Global Resonance, the relationship is reversed, with both top and bottom group males scoring higher than females. However, when males outscore females on individual scales, the difference between genders is much smaller than on the scales where females score higher.

Several other scales show no difference between females and males in the top group, although females score higher than males in the bottom group in scales such as Needs Fulfilment, Self Awareness and Sociocultural Openness. Importantly, the Identity Diffusion scale contrast in Fig. 6.3 shows distinctive differences between the top and bottom 30 percentages in each gender; although comparing the genders, the 
scores between the top 30\% males and top 30\% females are very close, and the scores between the bottom $30 \%$ males and $30 \%$ females are identical. Overall, there is no meaningful difference between male and female groups in relation to the Identity Diffusion scale. In short, for this sample, gender does not appear to be a strong differential predictor of Identity Diffusion, although gender is a strong predictor of global identity as measured by the Full Scale score (see Table 6.2).

\section{Aggregate Profile by Country of Origin}

Aggregate Profile by Country of Origin provides a comparison of how US and nonUS groups differ at the top and bottom $30 \%$ of this sample. For many scales, both top and bottom groups of participants raised in the USA and elsewhere are very similar (e.g. Needs Fulfilment, Socioemotional Convergence and Self Awareness). There are a few scales where non-US origin participants scored substantially higher only or especially in the bottom group. Some of these scales, such as Basic Determinism, are related negatively to the Full Scale score, while others, such as Sociocultural Openness and Ecological Resonance, contribute to the Full Scale score in a positive direction. Evidence from Fig. 6.4 suggests that participants of non-US origin are more likely than those from the USA to be open to culturally different others, and engaged and invested in environmental issues, regardless of the extent of their global identity as measured by the Full Scale score. On the other hand, the US origin group is less likely, across the board, to engage in black and white thinking and simple causal attributions.

The comparison for the Identity Diffusion Scale shows that there is minimum difference between the top and bottom $30 \%$ of the US group (16 and 22, respectively). However, the scores of non-US participants in the top and bottom groups are substantially different, which suggests an important finding vis-a-vis global identity. In particular, these results suggest that low Full Scale scorers who were raised outside the USA are experiencing a substantially higher degree of Identity Diffusion than any of the other three groups: US high Full Scale scores, US low Full Scale scores and Non-US high Full Scale scores. As such, a preliminary interpretation of these findings is that a greater degree of Identity Diffusion is mediated (i.e. influenced) by the scales/variables that are integral to the Full Scale score. In other words, (a) having less of a capacity to experience and/or make sense of one's own emotional world (lower Emotional Attunement), (b) exhibiting more black and white attributions (i.e. causal explanations) about why humans do what they do (higher Basic Determinism), (c) being less inclined to reflect on one's own psychological processes (lower Self Awareness) and (d) demonstrating a greater tendency to engage in stereotypical thinking about "the other" (lower Sociocultural Openness) — which are all variables associated with a lower Full Scale score and thus, for our purposes, a lower degree of global identity - are, in fact, quite strongly associated with a greater degree of confusion about who one is and why one feels "trapped" in one's own life, especially when moderated by not having been raised in the USA. In other words, although this is subject to further inquiry, it may be that those individuals who are already struggling 


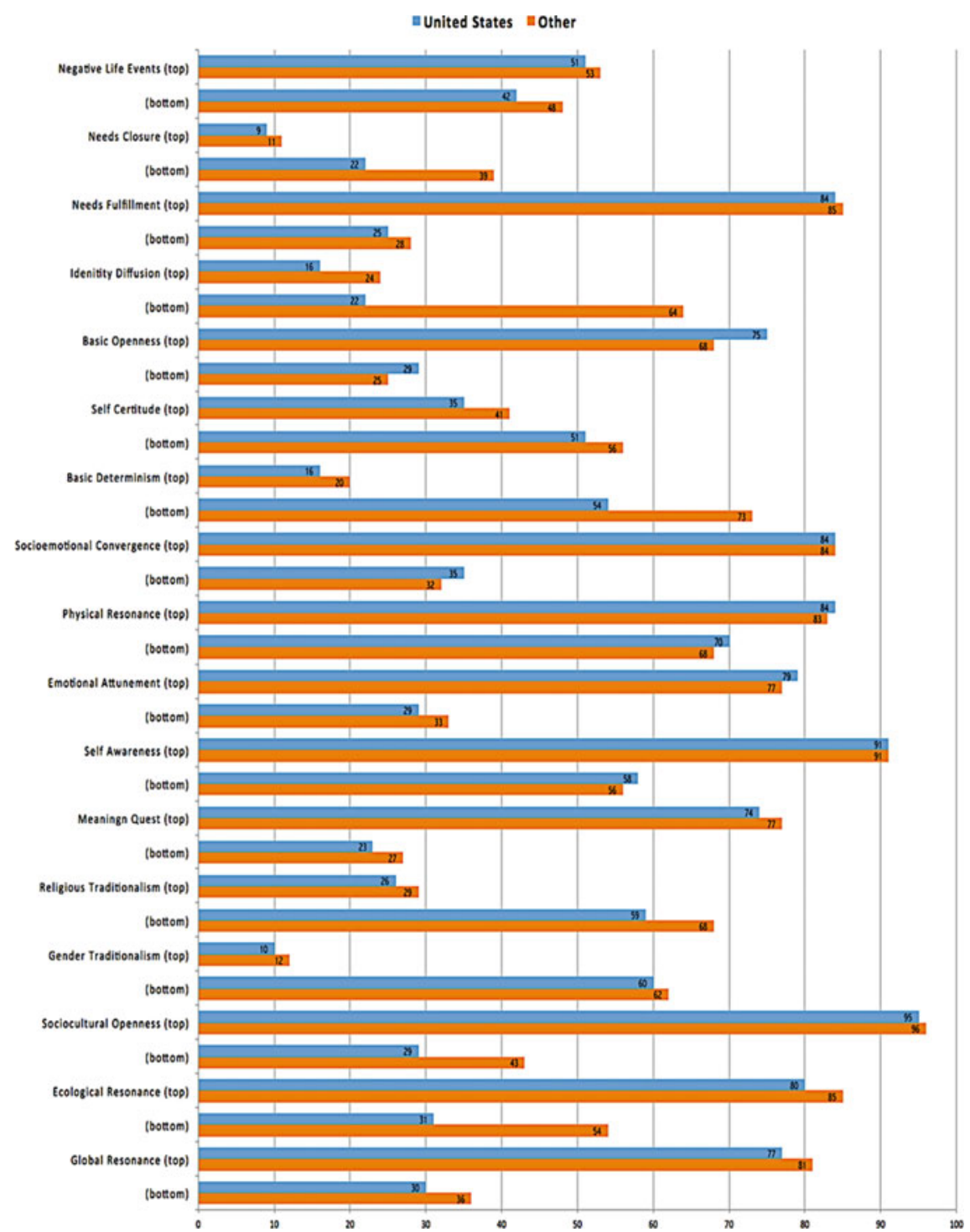

Fig. 6.4 Aggregate Profile — contrast by country of origin

with lower global identity in general, and a diffuse sense of identity in particular, are especially prone to the perception and/or reality that a new culture and context is disinclined towards them as "the other" (i.e. individuals who are "different" or "not from" the USA), with all of the attendant and negative implications in regard to their ability to assimilate and acculturate (e.g. to contribute to, and gain acceptance from, the new culture). 


\section{Aggregate Profile by Ethnicity}

Finally, as depicted in Fig. 6.5, Aggregate Profile by Ethnicity provides a comparison of Caucasian and non-Caucasian groups at the top and bottom $30 \%$ of this sample. Parallel to the country of origin comparison findings, we can see in Fig. 6.4 some scales showing little difference between the ethnic groups (e.g. Basic Openness and Socioemotional Convergence) and other scales where substantial differences do emerge. Again, as with country of origin groups, higher scores for non-Caucasian groups appear in both positive (e.g. Sociocultural Openness, Ecological Resonance, Global Resonance) and negative (e.g. Basic Determinism, Religious Traditionalism, Gender Traditionalism) directions with respect to the Full Scale score, which likely contributes to the results for ethnicity that we have seen in the Background-Domain Contrast (see Table 6.2).

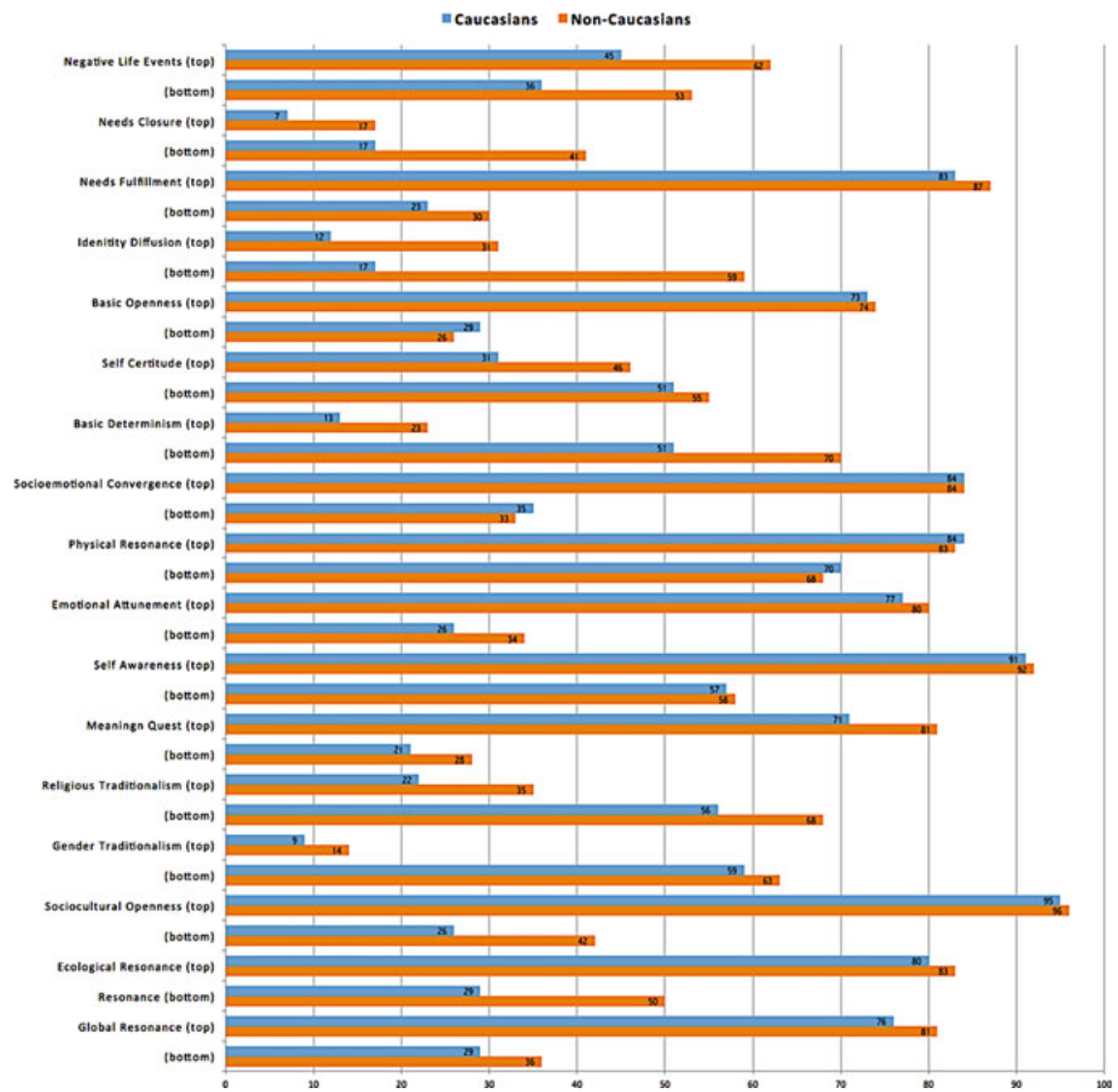

Fig. 6.5 Aggregate Profile—contrast by ethnicity 
Thus, we can conclude that while overall, Caucasians may be more likely to evince a higher global identity via their higher Full Scale scores, individual scale predictions do not always place Caucasians in this position. In fact, some of the most important scales that theoretically and statistically support global identification (i.e. Sociocultural Openness and Global Resonance) depict non-Caucasians as evidencing higher degrees of global identity, especially in the bottom $30 \%$ group.

Also, of particular note is the striking difference between Caucasians and nonCaucasians on the Negative Life Events scale, which suggests that the more disadvantaged populations (e.g. those more likely to experience prejudicial conduct or attitudes at home and abroad) may also report a greater degree of familial dysfunction, poverty, trauma, violence and other Negative Life Events. Along these lines, it is important to recognise that the gap for ethnicity at this level is substantially larger than that for the other moderating variables. Specifically, as was also evident on Aggregate Profile by Country of Origin (see Fig. 6.4), the Identity Diffusion comparison in Fig. 6.5 shows only a minimum difference between the top and bottom $30 \%$ within the Caucasian group (12 versus 17). In contrast, the dramatic differences for the nonCaucasian group, on both top (31\%) and bottom (59\%) groups for Identity Diffusion, suggest a powerful interaction between the moderator of ethnicity and the mediator of Identity Diffusion.

In this regard, and at a broader level, Negative Life Events is correlated with many of the scales that are used to calculate the Full Scale score, including Identity Diffusion with a relationship of 66 (Wandschneider et al., 2015). Stated differently, when participants have high Negative Life Events scores, they also tend to have higher Identity Diffusion scores, and vice versa. It therefore seems plausible that the negative experiences that non-Caucasians report subsequently influence their understanding and engagement with self, others and the larger world. These difficulties become magnified when one is low in global identity as operationalised here, for the same reasons as noted above in the observations about country of origin. That is, if an individual already has relatively less emotional and cognitive capacity including, but not limited to, lower Emotional Attunement and higher Basic Determinism-and is also non-Caucasian - they are much more likely to experience a sense of being lost, confused and trapped in their own lives and in the larger world, which is another important finding from the current analysis.

\section{Discussion}

The findings of this study inform best practices in culturally competent pedagogies and programmes - and by extension, policies — that seek to promote international, multicultural and transformational learning, growth and development. Among other implications, findings suggest that substantial differences exist within and between subgroups of a large and diverse sample of students, who engage in learning experiences that are designed to facilitate cultural competence through higher education. At this big picture level, we can learn much by understanding how and why our 
students become organised as they are, in terms of their sense of identity before, during and after we encounter them, and the applications of such understanding for the development, implementation and assessment of our programmes and courses.

As Spaeth et al. (2016) observe, identity "guides the formation of self-concept, and influences an individual's behaviour, cognitive capacities and emotional reactions" (p. 207). It thus behoves international education administrators, educators, practitioners, scholars and policy-makers to assess and apprehend how human identity both shapes and is shaped by international, multicultural and transformative learning experiences; as well as how, why and under what circumstances, these experiences contribute to greater-or lesser-culturally competent learning, growth and development. In short, by understanding better how our students experience themselves, others and the larger world, we will be better able to design, evaluate and improve pedagogies and interventions that meet those students where they actually are, rather than where we think they are or should be (Shealy, in press; Wandschneider et al., 2015).

\section{Student Readiness for International, Cross-Cultural and Transformative Learning}

At the most basic level of analysis, our results illustrate that at the beginning of their educational experiences, our students are very different people, exhibiting substantial variation in the ways "identity" and "self" are structured, from the standpoint of the BEVI method and EI model. We see this variation clearly in Aggregate Profile (see Fig. 6.4), which compares the top ("high optimal") and bottom ("low optimal") $30 \%$ of Full Scale score responders; this same pattern emerges in each subsequent Aggregate Profile that focuses on three moderators: gender, country of origin and ethnicity (see Figs. 6.3, 6.4 and 6.5).

\section{Moderators and Mediators of Global Identity}

Extending our analysis of Full Scale score results, we next considered global identity — which we have again operationalised via the BEVI Full Scale score-now juxtaposed with specific moderators and mediators. Recall that, from the Background-Domain Contrast (presented in Table 6.2), we discovered a clear trend for Full Scale scores with regard to the moderating variable of gender. In short, in this sample, females were more likely to have higher Full Scale scores than males, indicating a greater inclination towards the underlying precepts of "global identity" for females - a finding that is consistent with other research of this nature (Pendleton, Cochran, Kapadia, \& Iyer, 2016; Wandschneider et al., 2015). 
However, both females and males in the top groups evince much higher Emotional Attunement than those in the bottom groups; thus, while gender has emerged as an important moderator in this analysis, with females higher than males in both top and bottom groups, the same pattern we saw earlier in the EGA emerges again in this analysis for the Full Scale score. In other words, although females remain higher than males on Emotional Attunement in both the "high" and "low" optimal groupsan important finding in itself-these results illustrate what may be an even more intriguing observation: the basic affective/cognitive structures of global identity, as we define it here, appear to be a more powerful differentiator than the moderator of gender, in terms of what explains these differences. Therefore, how we are structured at these core levels of identity appears to be a more powerful predictor of these fundamental capacities (e.g. Emotional Attunement) than any of the moderating variables we examined in this study including, but not limited to, gender.

\section{Moderators and Mediators of Identity Diffusion}

We turn now from the holistic viewpoint of the macro-analysis to an examination of specific details, namely the Identity Diffusion Scale and its relationship to moderating and mediating variables. Focusing on this one scale accomplishes several purposes. Not only do the results of the Identity Diffusion Scale help confirm for us the validity of using the Full Scale scores as an indicator of the global identity construct, but we also gain insights into which subgroups are struggling most with their identities, at the point of entry into international and/or multicultural learning experiences. We know from other studies of T1/T2 BEVI data that mediating variables can change as a result of internationalised learning (Wandschneider et al., 2015) and that "change at the level of beliefs and values appears not only to occur and be measurable across time, but may show underlying patterns that help us evaluate the effectiveness of the intervention" (Shealy, 2016, p. 165).

Identity Diffusion is a particularly useful scale on the BEVI when it comes to formative assessment because it measures, among other factors, a participant's sense of discomfort with who they are, and lack of clarity about their place in the world. In this EGA analysis, participants as a whole have relatively low Identity Diffusion scores, with the bottom $30 \%$ group twice as high as the top $30 \%$ group, but still averaging in the lower third or so of the scale. While the gap for Identity Diffusion is not as large as we see with some other individual BEVI scales, it does still follow the typical pattern of data in this study, with a substantial top versus bottom group difference in the direction predicted by EI theory (Shealy, 2016).

It is important to note that the kind of anxiety reflected in the Identity Diffusion Scale is not necessarily harmful or disadvantageous, at least in the short term. Montuori and Fahim (2004) argue that, rather than necessarily a state to be avoided or eliminated, anxiety is "an integral part of being human" and "fundamentally, a source of learning and creativity" (p. 257). In other words, sometimes painful crises of identity may be a vital step towards greater self awareness and self-transformation. 
When we come into contact with worldviews, value systems and cultural practices that are strikingly different from-or even incompatible with-our own, we can be shaken to the core, since "our whole sense of making meaning of the world can literally be shattered" and "our confusion about the world will translate into a confusion about who we are" (p. 258).

There is a clear implication here for culturally responsive pedagogy and curriculum design: knowing that some students are coming to an experience already feeling a crisis of identity may well impact the level, extent and kinds of support that we should offer programme participants. Congruent with the above discussion, while some students may enter a learning experience in higher education ready to process it cognitively and affectively, others may need more preparation, reflection and support before, during and after the experience. Such perspective receives further and considerable additional support from a recent BEVI study of students from the Middle East, some of whom showed a number of similar findings to those obtained here (e.g. individuals with a lower capacity to access emotion, and higher tendency to make black and white attributions, struggled more with the high impact nature of the learning experience) (Giesing, 2017).

The highly intriguing findings from this study have yielded many implications and applications that are important for consideration by scholars, practitioners and leaders who are working to internationalise higher education, or who pursue greater effectiveness in international, multicultural and transformative education through evidencebased strategic planning, intentional curriculum design and culturally competent pedagogies. Drawing from the information discussed above, we have synthesised and compiled a list of 10 such implications and applications, as follows.

1. Learners in a large and diverse pretest sample exhibit very different levels of global identity, which in all likelihood impacts their learning experiences.

2. Both moderators (e.g. ethnicity) and mediators (e.g. Identity Diffusion) contribute to the development of global identity, but to different degrees for different subgroups.

3. It is necessary to examine both between-group and within-group differences (i.e. whatever measures one uses for assessment purposes, it is important to apprehend how similar or different individual members of groups are from each other).

4. Relatedly, aggregate scores tell an important story but not the whole story. Single aggregate measures like the BEVI Full Scale score can be very useful as general indicators of constructs like global identity, but important details may be missed if we do not go beyond a composite score (e.g. through "Decile Profile" and "Aggregate Profile By" indices).

5. Before we learn about others and the larger world, it helps to learn about self. Specifically, results suggest substantial differences regarding who we are as human beings (e.g. how do emotions work, how sophisticated are our attributional systems) which are strongly related to differences in global identity, as defined in this study. The benefits of spending time focusing on such matters in class or programmes are many (Iyer, 2013). In addition, individual BEVI 
reports may help participants acknowledge and reflect on their own structures of values and beliefs, whereas group report debriefings of BEVI results can help students better understand who they are in relation to their cohort, as well as what events, people and societal forces may have shaped who they have become (Wandschneider et al., 2015).

6. In addition to knowledge and skills, internationalised learning experiences must include attention to beliefs, values, experience and emotions. Students need to develop in all of these areas in order to achieve learning outcomes, such as increased intercultural competence (which encompasses the attitudes of openness and willingness to engage with other cultures), and leadership skills (which include greater self/other awareness and emotional intelligence).

7. Some participants will likely need more support than others (e.g. from findings like these, it is important to recognise that some students are likely to thrive and some to struggle, which indicates that we should create spaces and processes to anticipate and respond to such variation - through group meetings, individual check-ins, journalling and so on).

8. By learning about our students, we can evaluate the effectiveness of our recruitment and retention strategies. Some students are going to be more or less inclined to participate in international and cross-cultural experiences for reasons that may not be clear to them, or us, so if we want to reach the widest range of students, we may have to create opportunities for those who are less inclined to participate, to help them feel more comfortable doing so.

9. Although these results do suggest overarching findings that are applicable to averaged groups (e.g. non-US, non-Caucasian students), there is a large amount of within-group variability. As such, we should avoid stereotyping individuals or groups on the basis of attributions that are assumed to apply to all members of specific groups (e.g. gender, ethnicity, nationality, education, class), as our data indicate that such assumptions may be completely wrong.

10. Effective courses and programmes strive to engage and apprehend our students deeply (e.g. how and why they experience self, others and the larger world as they do). Such understanding has great potential for many "teachable moments" in the classroom and the real world. For example, the fact that the Full Scale score is a more powerful predictor overall than gender, ethnicity and country of origin suggests that our core sense of identity - "how our beliefs and values exist in the service of core human need" and "why we become whom we become"is more important than any singular personal characteristic, in terms of our inclination to listen to, and learn from, one another. Findings such as these can help generate reflective light rather than destructive heat, in discussing the most contentious and polarising issues of our day. 


\section{Why We Are Who We Are: Concluding Implications and Applications}

We undertook this research project with the intention to understand better how gender, ethnicity and country of origin function as moderators of the development of global identity, as measured by the BEVI, while also illuminating how various BEVI scales mediate identity for the subgroups of participants created by these variables. As this study demonstrates, the BEVI offers a sort of global identity "selfie" of a specific group when moderating and mediating variables are explored together. Such a snapshot is of great value, in examining not only a cross-sectional view of within-group differences, but also longitudinal studies that show how people change from one "selfie" to the next, perhaps in response to an international, multicultural or transformative learning experience (Wandschneider et al., 2015).

There are limitations to this study, including our focus on college-aged students, usage of Time 1 as opposed to T1/T2 data and binary distinctions between constructs such as gender. Likewise, although quite striking differences emerged between groups based upon the selected analytic variables, it would be necessary to replicate findings with an entirely new sample to ascertain the generalisability of these within- and between-group distinctions. Similarly, a cautionary note also applies to comparisons between different groups; for example, it would not be appropriate to conclude from these findings that one group is "superior" to another (e.g. Caucasian versus non-Caucasian; USA versus non-USA), a point that is explicated further below. On the contrary, what ought to be inferred from these results is that adverse circumstances, in any culture or context, may be associated with a greater struggle to apprehend and navigate one's own life journey. This working hypothesis should lead to empathy rather than stereotyping, as well as an appreciation for how our relative degree of privilege can fundamentally affect how, and why, we humans experience self, others and the larger world, as we do. A similar caveat applies to the interpretation of scale score change in general; as well as what we have learned about the nature of belief/value structures and interactions (e.g. high Religious Traditionalism does not necessarily mean low Emotional Attunement), the relationship of core needs to human values and the processes by which identity changes over time (Shealy, 2016).

Along these lines, it should be emphasised that the BEVI seeks to open spaces through which the measurable human impact of such processes (e.g. engaging beliefs, cultures and practices that are different from our own) can be experienced, understood and evaluated in a pluralistic, credible and ecologically valid manner. To understand this commitment, it may be helpful to reflect briefly on some relevant conceptual and methodological considerations. Much quantitative research of this nature relies on categorical (often binary) variables because that is what is required for most inferential statistical analyses; for example, over the years, BEVI researchers have examined a number of structural equation models that specify the "model"- the theories or concepts in our heads about how "reality" works-which is then subject to the "structural equation" statistical analysis. Basically, structural equation modelling (SEM) allows us to examine the empirical relationship among psychometrically measurable 
constructs (e.g. latent variables that meet appropriate standards of reliability and validity), which help explain the underlying relationships between predictors and criteria (Piotr, 2017). Using SEM with the BEVI, for example, we know that there is a relationship between moderating variables like ethnicity and mediating variables like Sociocultural Openness on the BEVI and that they interact together and culminate in differences among predictor/outcome variables, such as interest in, or satisfaction from, study abroad. Such SEM findings make sense given that overall, people who self-report as "White" or "Caucasian" tend to be lower on Sociocultural Openness than people who self-report as non-White (a finding that makes sense, since minority groups tend to know more about majority groups than majority groups know about minority groups, this being a matter of cultural adaptation and survival) (Tabit et al., 2016).

While such results are very intriguing, the story is even more complicated and interesting when we see such processes through the lens of SEM. More specifically, the higher one's Sociocultural Openness on the BEVI, the more interested one tends to be in the prospect of participating in study abroad, a result that is influenced by life history. This is because a greater degree of Negative Life Events, another scale on the BEVI, is associated with lower Sociocultural Openness, which also tends to be associated with lower interest in international/multicultural education in general (Tabit et al., 2016). The interactive implications and applications of these processes are considerable when one simultaneously examines how "formative variables" (e.g. life history, ethnicity, gender, language, education) are empirically associated with other scales on the BEVI, which further predict specific observable or outcomes variables (e.g. careers, grades, political orientation, religious commitments) (Acheson et al., in press; Shealy, 2016). Ultimately, to examine and understand these phenomena, which appear across cultures and contexts, we must have a way-statistically — to categorise differences between groups, even though we all recognise that such categorical variables have limitations. That is one reason why the BEVI is also a mixed-methods measure and includes qualitative in addition to quantitative questions (e.g. qualitative findings help us interpret and give texture, whereas quantitative findings give us precision, as well as the ability to make reliable and longitudinal comparisons across groups and experiences) (Iyer, 2013; Wandschneider et al., 2015).

At the same time, one of the most powerfully consistent findings from crosscountry/cross-cultural work with the BEVI is that there is greater within- than between-group variability. That is why it is extremely important not to stereotype any group_-including, but by no means limited to, White or non-White or western and non-western people, and people with any characteristics with which we identify (e.g. gender, politics, religion) - mainly because there is more variability within different groups of people than between them. In other words, from BEVI data across a very wide range of contexts and cultures, we repeatedly see that regardless of personal characteristics, histories or commitments, all human beings are completely capable of racism, sexism and every other "ism" across all demographic groups all over the world (e.g. see Pendleton, Cochran, Kapadia, \& Iyer, 2016; Tabit et al., 2016). Confusion around such matters (i.e. the belief that specific people from specific groups will be more_-or less_-racist, sexist, etc., than other people or groups)—particularly in 
public discourse or as a matter of policy (e.g. "White people are X", "Black people are Y", "males are X", "females are Y", etc.) — may be associated with and/or predictive of (1) casual to severe racism, sexism or even violent acts of aggression (e.g. mass shootings, sexual assault) and (2) harmful excesses of the "call out culture" (e.g. public shaming that leads to serious life and professional consequences without sufficient accountability, due process or self-reflection) (e.g. Aronson, 2012; Ross, 2019).

That is because from the standpoint of the BEVI, and its underlying EI theoretical framework, what we ultimately believe and value (a) is inextricably linked to how our core needs have been experienced by our caregivers and others throughout development, which (b) further shapes how our selves are structured (e.g. what we call good or true — or bad or false — about self, others and the world at large), which then (c) further influences why we think what we think, say what we say and do what we do (Shealy et al., 2012). Conceptually and empirically, this complex process by which we become who we are, and do what we do, may be statistically predicted, to a degree, by any number of variables (e.g. gender, ethnicity, country of origin) which do show-in the aggregate-trends such as those reported above (e.g. White people overall tend to be lower on Sociocultural Openness). However, again, BEVI data all over the world strongly indicate that there is more within-group than betweengroup difference. In other words, it is quite often the case that a White person will not show the characteristics of White people in general, males will not show the characteristics of males in general and so on. This is because we are enormously complex and multi-determined creatures and as such, singular variables — such as gender, ethnicity and country of origin-will indeed influence who we are and how we see self, others and the larger world, but only to a degree, and typically under specific circumstances which are mediated by other variables such as life history, education, political/religious background and so on (Pendleton et al., 2016; Shealy, 2016; Tabit et al., 2016).

Complicating matters further, abundant evidence from the BEVI also indicates that life history and events often have a profound effect on the manner in which those propensities are expressed and to what degree (e.g. how self, others and the larger world are experienced), a finding that emerged powerfully in the analyses reported above. For example, people who are experienced as "the other" by the dominant group in the USA often struggle more with issues of their own identity, particularly if they already were predisposed to do so via their own life history (which may have been rife with prejudice, struggle, etc.). Along these lines, as of this writing, there are over 60,000 administrations of the BEVI all over the world. From these findings, and hundreds of BEVI reports, it appears clear that one major task before us as a species is to learn how to regard "the other" with greater emotional and attributional complexity and care, and to recognise that we are all in this together (i.e. we all are capable of prejudice and delimiting beliefs and values about other human beings, processes which are mediated and moderated by the life experiences we have) (Shealy, in press). 
In short, such findings, including those reported in this chapter, should lead to compassion and understanding (e.g. about the multifaceted nature of "privilege" and the educational and policy implications of that) as we acknowledge the impact of life history/events on us and all the attendant applications; for example, we should take care of each other and recognise that the allocation of resources and the life histories we experience affect our ability to "adapt" to cultures that are different from our own. Illuminating those sorts of complexities is a major purpose of the BEVI, in addition to assisting allied work through the non-profit International Beliefs and Values Institute and Cultivating the Globally Sustainable Self Summit Series.

In the final analysis, we recognise the complexity of these interacting processes, which can make hard and fast determinations of "what works best" as international, multicultural and transformative educators challenging but also eminently doable, if our approaches to assessment-based intervention are sufficiently comprehensive, persistent and ecologically valid. Towards such means and ends, as was discussed above, in terms of the need for multifaceted measurement of global competence, hundreds of analyses suggest that there is no "one" scale that "works best" or "always shows" greater pre-/post-change. Space limitations prevent further discussion of alternative or complementary approaches to measurement, although many comparative sources are readily available (e.g. Roy, Wandschneider, \& Steglitz, 2014). What we have seen repeatedly is that change processes appear to be associated with specific characteristics of individuals and groups, which interact further with the type, quality and nature of the educational or programmatic intervention. This perspective has been described as the 7Ds of change (Wandschneider et al., 2015), which may be helpful in attending to the multiple factors that appear associated with international, multicultural and transformative learning.

In that regard, as long as the above complexities are kept in mind, the findings presented here offer important guidance for how we approach the development and delivery of educational programmes, practices and policies (e.g. the 10 implications and applications for learning, growth and development, listed above). For example, in working with emotionally-laden content, we have learned that process is as, if not more, important than content; that is, instructors are advised to spend sufficient time dealing with the emotional sequelae of presenting controversial issues to their students, rather than simply delivering factual content (Tabit et al., 2016). This study also illustrates how population variables that are typically collapsed, or not even examined, have powerful implications for the design, implementation and analysis of international, cross-cultural and transformative learning experiences (Acheson et al., in press; Goodman \& Gorski, 2015; Iyer, 2013).

By rigorously investigating such underlying processes through sophisticated and comprehensive measurement with an unusually large sample, we can see how moderating variables_-gender, ethnicity and country of origin_-interact with mediating variables-such as Identity Diffusion, Sociocultural Openness, Global Resonance and other individual BEVI scales - to shape the relative ability and willingness of students to learn, grow and develop as a result of international, multicultural and transformative educational experiences. Ultimately, this research offers a way to understand and evaluate who learns what, and why, and under what circumstances. 
Such information can help us develop, implement and evaluate more effective and meaningful programmes, processes and policies, while also reminding us about one of the fundamental and overarching purposes of education: to cultivate globally sustainable selves and the capacity to care for others and the world at large.

\section{References}

Acheson, K., Dirkx, J., Wiley, J., Ullum, C., Kapadia, S., \& Bhuyan, D. (in press). Operationalizing transformation of the self in high impact learning: Methodological considerations. In C. Shealy (Ed.), The globally sustainable self. Oxford: Oxford University Press.

Altbach, P. G., \& Knight, J. (2007). The internationalization of higher education: Motivations and realities. Journal of Studies in International Education, 11(3-4), 290-305. https://doi.org/10. $1177 / 1028315307303542$.

Aronson, E. (2012). The social animal (11th ed.). New York, NY: Worth Publishers.

Betancourt, H., \& López, S. R. (1993). The study of culture, ethnicity, and race in American psychology. American Psychologist, 48(6), 629-637. https://doi.org/10.1037/0003-066X. 48.6.629.

Brubaker, R., \& Cooper, F. (2000). Beyond "identity". Theory and Society, 29, 1-47. https://doi. org/10.1023/A:1007068714468.

Cortese, A. D. (2003). The critical role of higher education in creating a sustainable future. Planning for Higher Education, 31(3), 15-22.

Deardorff, D. K. (2016). Outcomes assessment in international education: Changing the paradigm. In E. Jones, et al. (Eds.), Global and local internationalization (pp. 83-89). Rotterdam: Sense.

Dolby, N. (2004). Encountering an American self: Study abroad and national identity. Comparative Education Review, 48(2), 150-173. https://doi.org/10.1086/382620.

Egan, S. K., \& Perry, D. G. (2001). Gender identity: A multidimensional analysis with implications for psychosocial adjustment. Developmental Psychology, 37(4), 451-463. https://doi.org/ 10.1037/0012-1649.37.4.451.

Ferrari, M., \& Sternberg, R. J. (Eds.). (1998). Self-awareness: Its nature and development. New York: Guilford Press.

Gacel-Ávila, J. (2005). The internationalisation of higher education: A paradigm for global citizenry. Journal of Studies in International Education, 9(2), 121-136. https://doi.org/10.1177/102831530 4263795.

Giesing, W. (2017). MEPI, BEVI, and EI leadership: Implications and applications for global leadership assessment and development (Unpublished doctoral dissertation). James Madison University, Harrisonburg, VA.

Goodman, R. D., \& Gorski, P. C. (Eds.). (2015). International and cultural psychology. Decolonizing "multicultural" counseling through social justice. New York, NY: Springer.

Harter, S. (1999). The construction of the self: A developmental perspective. New York, NY: The Guilford Press.

Hendershot, K., \& Sperandio, J. (2009). Study abroad and development of global citizen identity and cosmopolitan ideals in undergraduates. Current Issues in Comparative Education, 12(1), $45-55$.

Hudzik, J. K., \& Stohl, M. (2009). Modelling assessment of the outcomes and impacts of internationalisation. In H. de Wit (Ed.), Measuring success in the internationalisation of higher education (pp. 9-21). Amsterdam: European Association for International Education (EAIE).

Institute of International Education. (2018). Number of international students in the United States reaches new high of 1.09 million. Retrieved from https://www.iie.org/en/Why-IIE/Announcem ents/2018/11/2018-11-13-Number-of-International-Students-Reaches-New-High. 
Iyer, C. (2013). Assessing and engaging beliefs and values in a learning community of U.S. and international students: Implications and applications from the Forum BEVI Project (Unpublished doctoral dissertation). James Madison University, Harrisonburg, VA.

Keeling, R. (2006). The bologna process and the Lisbon research agenda: The European Commission's expanding role in higher education discourse. European Journal of Education, 41(2), 203-223.

Kehl, K., \& Morris, J. (2008). Differences in global-mindedness between short-term and semesterlong study abroad participants at selected private universities. Frontiers: The Interdisciplinary Journal of Study Abroad, 15, 67-79.

Kim, E. (2012). An alternative theoretical model: Examining psychosocial identity development of international students in the U.S. College Student Journal, 46(1), 99-113.

Leary, M. R., \& Tangney, J. P. (Eds.). (2012). Handbook of self and identity (2nd ed.). New York, NY: Guilford Press.

Mendoza, S. L., Halualiani, R. T., \& Drzewiecka, J. A. (2002). Moving the discourse on identities in intercultural communication: Structure, culture, and resignifications. Communication Quarterly, 50(3-4), 312-327. https://doi.org/10.1080/01463370209385666.

Mezirow, J. (1997). Transformative learning: Theory to practice. In P. Cranton (Ed.) Transformative learning in action: Insights from practice, New Directions for Adult and Continuing Education, No. 74, 5-12. San Francisco: Jossey-Bass.

Montuori, A., \& Fahim, U. (2004). Cross-cultural encounter as an opportunity for personal growth. Journal of Humanistic Psychology, 44(2), 243-265. https://doi.org/10.1177/0022167804263414.

Pendleton, C., Cochran, S., Kapadia, S., \& Iyer, C. (2016). Understanding the gendered self: Implications from EI Theory, the EI Self, and the BEVI. In C. N. Shealy (Ed.), Making sense of beliefs and values (pp. 261-302). New York, NY: Springer Publishing.

Piotr, T. (2017). An overview of structural equation modelling: Its beginnings, historical development, usefulness and controversies in the social sciences. Quality \& Quantity, 52(1), 313-354.

Preacher, K., Rucker, D., MacCallum, R., \& Nicewander, A. (2005). Use of the extreme groups approach: A Critical re-examination and new recommendations. Psychological Methods, 10(2), 178-192. https://doi.org/10.1037/1082-989X.10.2.178.

Roy, P., Wandschneider, E., \& Steglitz, I. (2014). Assessing Education Abroad Outcomes: A Review of the BEVI, IDI, and GPI. White Paper. East Lansing: Michigan State University Office of Study Abroad. Retrieved from https://educationabroad.isp.msu.edu/files/2914/9486/1612/Assess ing_EA_Outcomes_WhitePaper.pdf.

Ross, L. (2019, August 17). I'm a black feminist. I think call-out culture is toxic. There are better ways of doing social justice work. The New York Times. Retrieved from https://www.nytimes. com/2019/08/17/opinion/sunday/cancel-culture-call-out.html.

Trooboff, S., Vande Berg, M., \& Rayman, J. (2008). Employer attitudes toward study abroad. Frontiers: The Interdisciplinary Journal of Study Abroad, 15, 17-33. Retrieved from http://files. eric.ed.gov/fulltext/EJ878375.pdf.

Sawir, E. (2013). Internationalisation of higher education curriculum: the contribution of international students. Globalisation, Societies and Education, 11(3), 359-378. https://doi.org/10.1080/ 14767724.2012 .750477$.

Shealy, C. N. (2005). Justifying the justification hypothesis: Scientific-humanism, Equilintegration (EI) Theory, and the Beliefs, Events, and Values Inventory (BEVI). [Special Series]. Journal of Clinical Psychology, 61(1), 81-106.

Shealy, C. N. (Ed.). (2016). Making sense of beliefs and values: Theory, research, and practice. New York, NY: Springer.

Shealy, C. (Ed.) (in press). Cultivating the globally sustainable self: How the human species might fulfil its potential. Oxford, England: Oxford University Press.

Shealy, C. N., Bhuyan, D., \& Sternberger, L. G. (2012). Cultivating the capacity to care in children and youth: Implications from EI theory, EI self, and BEVI. In U. Nayar (Ed.), Child and adolescent mental health (pp. 240-255). New Delhi, India: SAGE. 
Spaeth, J., Schwartz, S., Nayar, U., \& Ma, W. (2016). Identity development and the construction of self: Findings and implications from the forum BEVI project. In C. Shealy (Ed.), making sense of beliefs and values: Theory, research, and practice (pp. 205-231). New York: Springer.

Tabit, M., Legault, L., Ma, W., \& Wan, K. P. (2016). In search of best practices for multicultural education: Empirical evidence from the forum BEVI project. In C. N. Shealy (Ed.), Making sense of beliefs and values: Theory, research, and practice (pp. 177-203). New York, NY: Springer.

Taylor, E. W., \& Cranton, (Eds.). (2012). The handbook of transformative learning: Theory, research, and practice. San Francisco: Jossey-Bass.

Wandschneider, E., Pysarchik, D. T., Sternberger, L. G., Ma, W., Acheson, K., Baltensperger, B., et al. (2015). The Forum BEVI project: Applications and implications for international, multicultural, and transformative learning. Frontiers: The Interdisciplinary Journal of Study Abroad, $25,150-228$.

Felix Wang serves as the Associate Executive Director for the Center for Global Engagement at James Madison University. He earned his Master of Business Administration from Campbell University, Master of Education in College Student Personnel Administration from James Madison University, and $\mathrm{PhD}$ at the Center for Higher Education Internationalisation (CHEI), Università Cattolica del Sacro Cuore. Felix has over 15 years' experience in the field of international education.

Kathleen Pait is a student in the Combined-Integrated Doctoral Program in Clinical and School Psychology at James Madison University. Her research interests include the psychology of religion, spirituality and identity. She has taught an undergraduate course entitled "Making Sense of Beliefs and Values: A Guided Tour for Global Citizens." She holds a Master of Arts in Psychology in Education from Columbia University.

Kris Acheson (Ph.D., Arizona State University, 2008), a former Fulbright scholar to Honduras, currently serves as director of Purdue University's Center for Intercultural Learning, Mentorship, Assessment and Research, with a courtesy Faculty appointment in the Brian Lamb School of Communication. Her research interests include the development and assessment of intercultural competence in higher education.

Lee Sternberger is Executive Director of the Center for Global Engagement at James Madison University, leading the Centre's ongoing process of internationalisation. She holds a $\mathrm{PhD}$ in clinical psychology from Washington State University and a Master of Arts in Architectural History from the University of Virginia. Lee's research focuses on the assessment of international, multicultural, and transformative learning.

Renee Staton is Professor in the Department of Graduate Psychology's Counselling Programs, at James Madison University. Her research interests include multicultural counselling, international education, and gender issues in counselling. Her recent work includes an exploration of counselling needs of students who are immigrants, and consultation regarding cross-cultural and ethical issues in counselling and agency settings.

Craig N. Shealy is Executive Director of the International Beliefs and Values Institute and Professor of Graduate Psychology at James Madison University. His publications include Cultivating the Globally Sustainable Self, (forthcoming, Oxford University Press) and Making Sense of Beliefs and Values (2016, Springer Publishing). Dr Shealy is an American Psychological Association (APA) Fellow and Past President of APA's Division of International Psychology. 
Open Access This chapter is licensed under the terms of the Creative Commons Attribution 4.0 International License (http://creativecommons.org/licenses/by/4.0/), which permits use, sharing, adaptation, distribution and reproduction in any medium or format, as long as you give appropriate credit to the original author(s) and the source, provide a link to the Creative Commons license and indicate if changes were made.

The images or other third party material in this chapter are included in the chapter's Creative Commons license, unless indicated otherwise in a credit line to the material. If material is not included in the chapter's Creative Commons license and your intended use is not permitted by statutory regulation or exceeds the permitted use, you will need to obtain permission directly from the copyright holder.

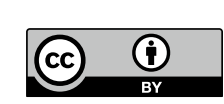

